\title{
A Control Strategy of a Haptic Display Using Honeycomb
}

\author{
Ryota ISHIBASHI, * \\ ${ }^{1}$ Tokyo Metropolitan University, 6-6 Asahigaoka Hino Tokyo, Japan \\ ${ }^{*}$ Corresponding author
}

Keywords: Impedance control, Perception, Tactile display, Honeycomb structure, Graph theory, Group, Lie bracket.

\begin{abstract}
This paper proposes a kind of tactile display using composite materials. Proposed display consists of a core honeycomb structure and a bi-elastic material. The system has both the endoskeltal and the exoskeltal properties. Then, it can realize an elastic display strategy for rendering softness based on these flexible materials. In the system, pressed by an actuator, the display is stretched with its apparent stiffness changed. The internal honeycomb structure ensures that the display has the same stiffness value everywhere on its surface. Thus, we can control the stiffness of the display only with one actuator. At first, the structure and the control strategy of the display was expressed. Then, basic principal of the tactile display was examined. Some experiments were conducted to verify the effectiveness of the proposed display. In addition, robustness of the material structure was examined.
\end{abstract}

\section{Introduction}

Softness display technology has been developed to various fields, such as virtual reality systems, touch screen systems, or teleportation systems [1]-[4]. Especially in the medical applications, softness displays have received a lot of attention that it can realize tactile sensation of the physiological tissue for the doctors virtually. In order to develop more effective sensation, we need some mechanical force transfer methods. Methods using robot manipulators use the end point of the manipulator, such as the PHANToM. On the other hands, touch-screen type display systems were developed to realize a direct touch tactile display. This kind of devices can display tactile information without mechanical force transfer; the dynamics of the mechanism of the presentation in the device exert little influence on the presented information. In addition, for the medical use, the operator can experience the tactile characteristic of tissue by directly touching the surface of the display.

The touch-screen type Softness display usually developed to realize both the physical softness and the visual texture [5]-[14]. In one method, an array of pins arranged under a flexible sheet is rapidly adjusted soon by actuator movement, and the elasticity distribution is expressed on a flexible sheet. To express spatially broad distribution of very minute elasticity, the number of pins and the actuator area correspond to the resolving power. In another method, the stiffness in the normal direction is tuned by adjusting the bias tension of a flexible sheet. In the method, the stiffness of the sheet can be adjusted only with the actuator to express the tension. Controller will calculate and assist the stiffness in the normal direction which has a different value in each part of the sheet under a constant bias tension. A new softness display is therefore examined, which proposes control based on the contact area of the tip of a finger, which provides changeability in the method $[12,13]$. In the material approach, a method of adjusting both shape and the elasticity of the display is proposed 
by using inverted pseudo plastic fluid. A passive element with variable mechanical impedance for wearable robots was proposed $[14,15]$. Related study provided effective the display method [1]-[17].

If we realize these effects by using more simple structure, we can realize useful display to show the visual and elastic information. In addition, the material is not physically restrained, and because a passive element is used for the display, operator safety is easily ensured. Passive viscoelastic material provides the ability to express particular textures not easily evaluated by a single index. However, to provide a sufficiently large display area while considering its ease of use, the driving force for the response must be minimized. The same dimensions of the display area as those of the displayed tissue will be needed to improve the presentation in the future. In addition, it is necessary to be able to mechanically construct it in a simple structure.

In this research, we propose a method to attain a sufficiently large display area to represent desired softness. Fig. 1 shows the proposed display material. The proposed display is constructed by a viscoelastic fluid resin and a core material. The internal core material formed a kind mesh structure. The actuator changes its horizontal special direction, and the internally caused stress is changed because the fluid is able to expand and contract. The stiffness in the vertical special direction is adjusted by changing the residual stress. Thus, the tactile feel can be adjusted.

Finger tip

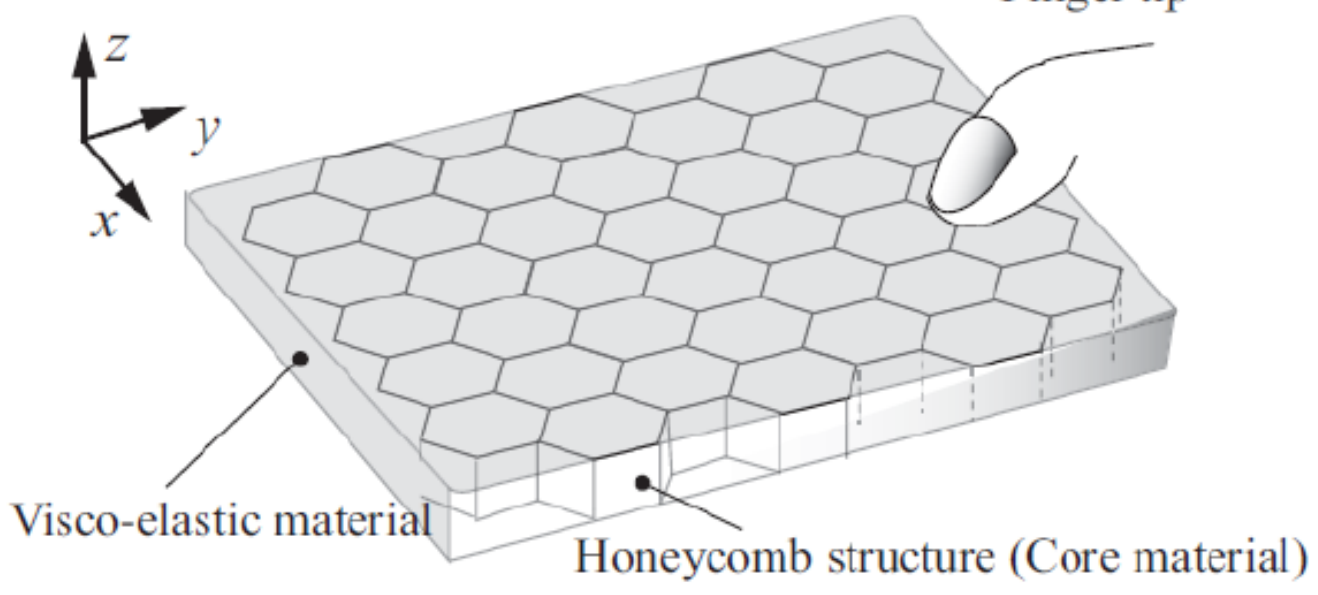

(i) Proposed display material

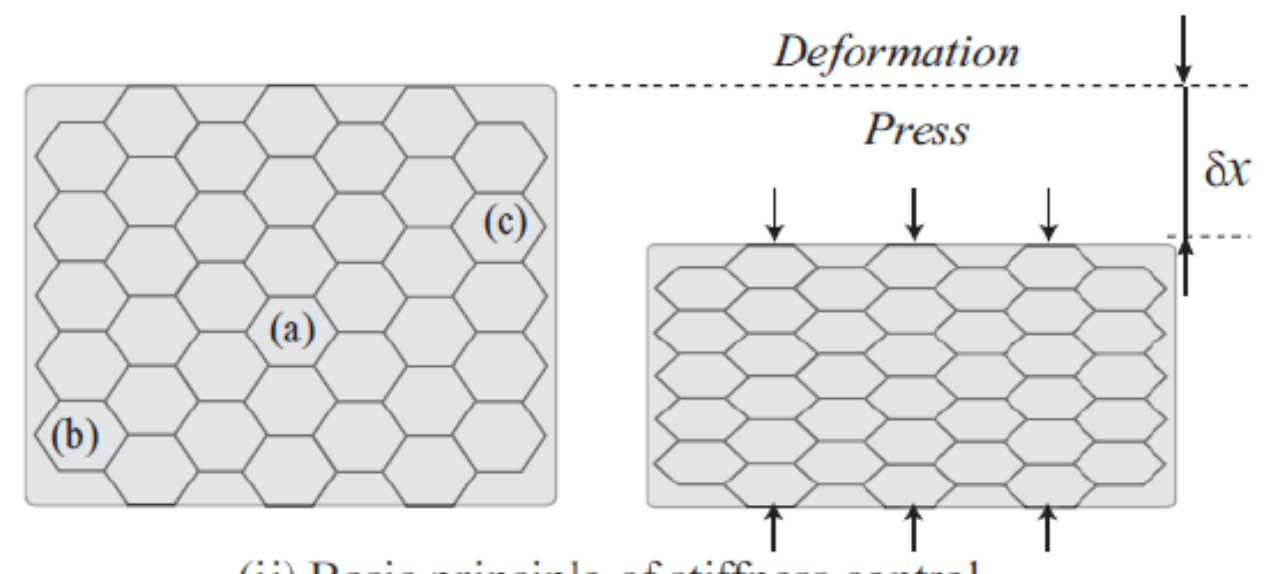

(ii) Basic principle of stiffness control

Figure 1. Sketch of the proposed material 
In this principle, the stiffness is simply adjusted by the expansion and contraction of the display. The stiffness is the same at each part of the display area due to the core material of the mesh being evenly distributed in the display material. The material is not physically restrained, and because a passive element is used for the presentation device, operator safety is easily ensured. In addition, image projection from the rear can also be enabled. Thus, it becomes important to design the location of the core material of the mesh.

In this paper, firstly introduce a basic principal of a proposed softness adjustment method and then the effects of the softness adjustment is verified by some experimental results.

\section{Basic Principal of the Display}

This section shows a basic principal of the proposed haptic display structure.

\section{Shape of the Internal Cell [19]}

This display controls the residual stress by the expansion and contraction of the direction of the $x$ axis shown in Fig. 1. Then, the sense of touch when touching from the direction of the $\mathrm{z}$ axis as face of a panel is adjusted. In general, it is difficult to change the residual stress of a flexible resin of plane externals thoroughly by expansion and contraction from the side. Additionally compressing it in excess too much causes the upheaval of the center part. Therefore, it is necessary to stabilize making of the stress distribution and uniform the structure by some methods. For the practical use, it should be better to realize a simple actuation for the stress control strategy.

In the proposed procedure, the problem is solved by putting the core material of the mesh in the resin. At this time, it is preferable that each hole of the reticulation is a similar figure. Moreover, the fewer the surface area of the reticulation seen on the presentation side is, the better. Then, our previously proposed study, we think about the problem as the tessellation problem of the hexagon cell geometry [19]. Here, we assume that the cell geometry be graph $G$, then neighborhood of the $N i$ of the $i$ th node point becomes as follows.

$$
N_{i}=\{j \mid j \in \nu(G):(i, j) \in \epsilon(G)\},
$$

Where, $v(G)$ and $\varepsilon(G)$ are finite set. $v(G)$ is vertex set and $\varepsilon(\mathrm{G})$ is edge set of the graph $\mathrm{G}$. Here, we assume that edge connectivity of the $i$ th node point as $Z i$. Zi be the average number of sides meeting at a vertex. Let $n$ be the average number of sides of a regular polygon, $n$ becomes

$$
n=\frac{2 Z_{i}}{Z_{i}-2} .
$$

Here we think about the problem is the tessellation of the regular polygon, $Z i$ and $n$ must be natural number. Then we obtain the combinations $\{n, Z i\}$ as $\{3,6\},\{4,4\},\{6$, $3\}$, for the tilings in the display. The edge of the honeycomb cell should be better not to affect softness perception with the fingertip contact area. Then, $\{6,3\}$ as the honeycomb of the hexagon minimizes the display surface area viewed along the $z$ axis. In addition, hexagon shape is the fewest the surface area on the display side, and it is suitable for the image presentation. 


\section{Deformation of the Haptic Display [19]}

Next, we think about the transformation by external force which acts to the structure. It is preferable that the number of actuators for expansion and contraction is little. In addition, it is preferable that the actuator to give the external force doesn't disturb the presentation of the visual information with the display. In this research, the external force is given from the side of the structure.

Here, regular polygon is assumed to be a closed linkage structure that has the resilient joint. Let $m$ be the degrees of freedom and we obtain the following equation.

$$
m=3(n-j-1)+\sum_{i=1}^{j} f_{i} .
$$

Here, $n$ is a number of links, $j$ is number of joints, and $f i$ is degrees of freedom of each joint. All the culminations are assumed to be a rotary joint of one DOF (degrees of freedom). Then, deformation degrees of the hexagon as combinations $\{6,3\}$ become three degrees of freedom. The compressive deformation that uses one vector becomes possible as shown in Fig. 1(b). In addition, the transformation into various shapes becomes possible by assuming the three degrees of freedom. It is possible to expand and contract in the direction of the $x$ axis and the direction of the $y$ axis when the core material is put internally like Fig. 1. Moreover, it has a high stiffness and it stabilizes structurally for the external force from the direction of the $z$ axis. Therefore, appropriate softness can be presented by combining with a flexible resin.

\section{Stiffness Adjustment}

This section shows a proposed stiffness adjustment principal of the proposed display.

\section{Compression of Single Cell}

Fig. 2 shows a single cell geometry filled urethane resin. When the displacement $\delta x$ will add to the cell, the volume becomes as follows:

$$
V=2 x h\left(l+\sqrt{l^{2}-x^{2}}\right)
$$

Where $x=x_{0}-\delta x / 2$ is the size of cell and $x_{0}$ is the initial value. $l$ is the length of the edge and $h$ is the thickness. Let $K$ be the volume elasticity modulus as

$$
K=-V\left(\frac{\partial p}{\partial V}\right)_{T} .
$$

From the Eq. (4) and the Eq. (5), we can see that $K$ can be controlled by the displacement $\delta x$. 
Urethane resin

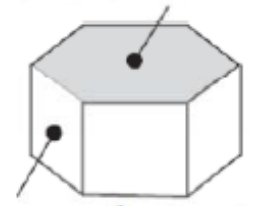

Honeycomb structure

(a) An internal cell
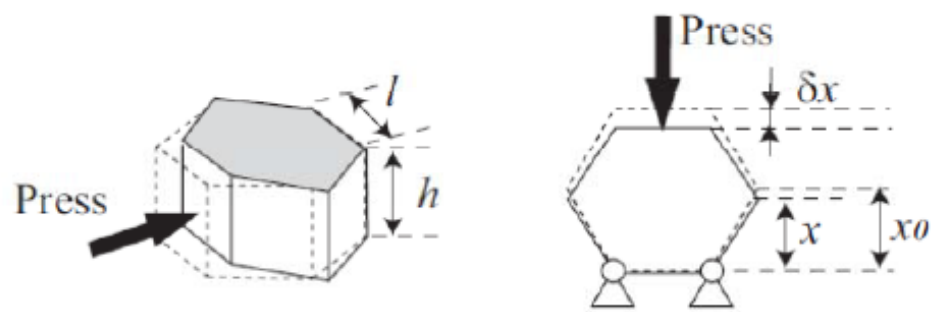

(b) Deformation of an internal cell

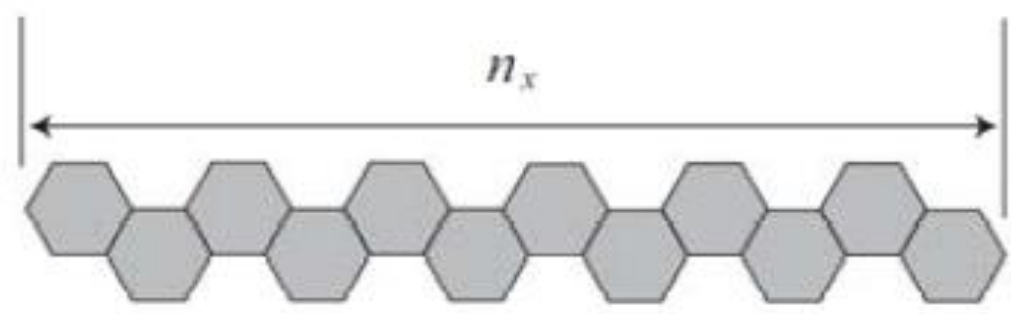

(a) Initial state

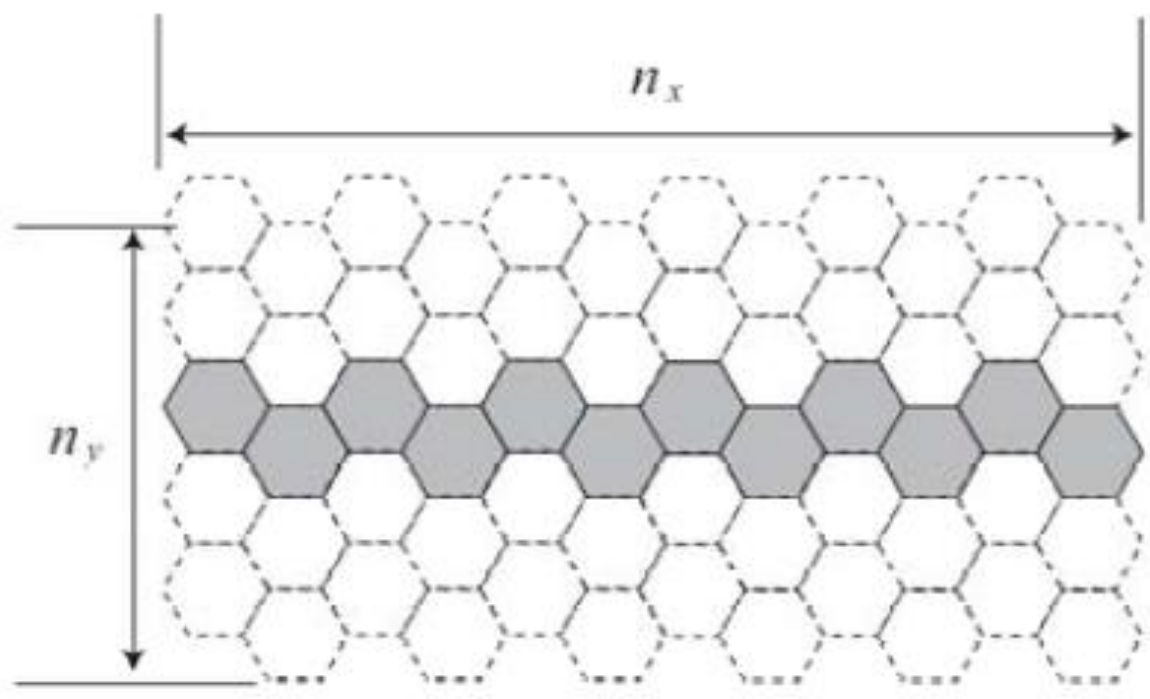

(b) Matrix form

Figure 3. Model of a cell matrix

\section{Compression of Cell Matrix and Stiffness}

Fig. 3 shows a model of the cell matrix set $\Delta$ with $m$ cells. Here we simplify the tessellation problem. Fig. 3 (a) shows a standard row where cells are connected to construct a line. Then the cell matrix will be constructed to make a rectangle like shape (the $n_{x}$ column and the $n_{y}$ row). $\mathrm{S}_{\mathrm{i}}$ is the subset of the set $\Delta$ and it can be inductively defined as follows. 
(1) $S_{1}=\left\{a_{1}\right\}, \quad\left\{a_{1}\right\}$ is the arbitrary member of the set $\Delta$

(2) $S_{2}=\left\{a_{1}, a_{2}\right\}, \quad\left\{a_{1}\right\}$ and $\left\{a_{2}\right\}$ share one edge

(3) If $2 \leq i$ and $S_{i}=\left\{a_{1}, a_{2}, \ldots, a_{i}\right\}$ can be defined, $S_{i+1}=\left\{a_{1}, a_{2}, \ldots, a_{i}, a_{i+1}\right\} 1 \leq h \in Z<k \in Z \leq i$,

(i) $a_{h}, a_{k} \in S_{i}$

(ii) $\left\{a_{h}, a_{h+1}\right\},\left\{a_{k}, a_{k+1}\right\}$ share one edge

Let $N_{i}$ be the set of node points $j$ at most near the point $i$ other than $i$. Then $N_{i}$ becomes adjacent points of $i$ and the number of $N_{i}$ becomes 2 or 3 . Let we call the vertex of $S_{n}$ satisfies $N_{i}=3$ as the good-vertex, and let the detail of such the good-vertex be $\lambda_{S n}$. Then we call the edge $\varepsilon_{i, j}$ of the $S_{\mathrm{n}}$ consists of the good-vertex $i$ as good-edge, and let the detail of such the good-edge be $\Gamma_{S n}$. Here we call such a set $S_{\mathrm{n}}$ as magic-triangle, and then, the number of edges consists of vertex of $S_{\mathrm{n}}$ satisfies $N_{i}=3$ becomes $4 m$.

Here we assume that the edges as the rigid linkage structures and the nodes as the rotary joints. When the displacement $\delta x$ will add to the cell matrix, the volume becomes as follows:

$$
V_{n}=2 m x h\left(l+\sqrt{l^{2}-x^{2}}\right) .
$$

From the Eq. (5) and the Eq. (6), we can see that the stiffness of the cell matrix can be controlled by the displacement $\delta x$.

\section{Stiffness Adjustment}

In the proposed display, we compress the display material from the side and adjust the stiffness. Then, it becomes important to realize stiffness adjustment method. Related study proposed a candidate of the controller to obtain the anti-resonance condition [17] [18]. Using these methods, this paper uses a controller to obtain the resonance condition for the haptic display to oscillate the display.

Here, we assume that the displacement $u$ is added to the display from the side.

$$
m \delta \ddot{x}=-d \delta \dot{x}-k_{1} \delta x-k_{2}(\delta x-u)
$$

Where $m$ is a mass of the display and $d$ is a viscosity.

$$
u=A \sin (\omega t)
$$

We add a control input to the system. Common case, we set the parameters amplitude $A$ and frequency $\omega$ in order to compress the display material.

In additional case, if we want to make a vibration display with the frequency $\omega$, we adjust the stiffness $k_{1}$ by using a following stiffness adjustment method to realize the resonance condition.

$$
k_{1}=k_{0}+\gamma \int_{0}^{t}\left(\delta \dot{x}(\tau)-\frac{k_{2}}{d} u(\tau)\right) \delta x(\tau) \mathrm{d} \tau
$$

Where, $k_{0}$ is an initial value of $k_{1}$ and $\gamma$ is a gain of this stiffness adjustment method. This method can adjust the stiffness $k_{1}$ to realize the system to be resonance condition 
with the frequency $\omega=\left\{\left(k_{1}+k_{2}\right) / \mathrm{m}\right\}^{1 / 2}$. Then, to investigate the stability of this controller, we use a candidate of the Lyapunov function as follows [21].

$$
\begin{aligned}
W & =W_{1}+\frac{1}{\gamma}\left\{\left(k_{1}+k_{2}\right)-m \omega\right\}^{2} \\
W_{1} & =m\left(\delta \dot{x}-\frac{k_{2} A}{d} \sin \omega t\right)^{2} \\
& +m \omega^{2}\left(\delta x+\frac{k_{2} A}{d \omega} \cos \omega t\right)^{2}
\end{aligned}
$$

Thus, we obtain $W \geq 0$ and the time derivative of the Lyapunov function becomes, $\frac{\mathrm{d} W}{\mathrm{~d} t}=-2 d\left(\delta \dot{x}-\frac{k_{2} A}{d} \sin \omega t\right)^{2}$ and then, we obtain the relation time goes infinity.

$$
\dot{W} \leq 0 \text {, when } k_{1}+k_{2}=m \omega^{2},
$$

Thus, we can oscillate the display at the desired frequency $\omega$. The controller looks like a synchronization of the parametron circuits but different.

Proposed controller uses an adaptive controller and the resonant condition as the desired state. In addition, it is unnecessary to satisfy such as the PE condition.

Thus, we can oscillate the display for the various desired frequencies. In addition, we can estimate the stiffness of the display materials instead of using nominal values.

\section{Experimental Results}

In the proposal device, the stiffness is adjusted by expanding and contracting the resin. To confirm the effect of this proposal principle, the base was examined.

\section{The Display Material}

At first, we explain the basic property of the display material. Fig. 4 shows an experimental system with the urethane resin and the honeycomb made of aluminum core material (cell size $9.0[\mathrm{~mm}]$ ). At this time, we control the distance along $x$ axis of the material and try to investigate how the stiffness along $z$ axis will be changed. Fig. 5 shows the stiffness change when assuming $0.0[\mathrm{~mm}]$ to $12.0[\mathrm{~mm}]$.

Here, it can be confirmed that the stiffness value has changed by compressing $x$ axially. Moreover, it can be confirmed that the deeper amount $z$ of pushing is, the bigger the stiffness change is. 


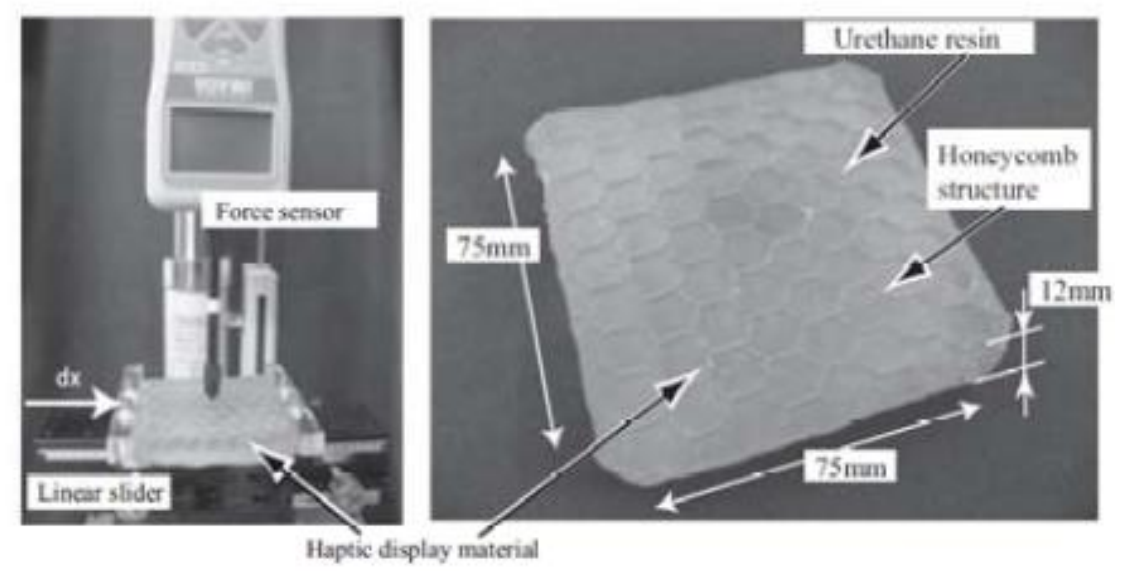

Figure 4. An experimental system and a display material

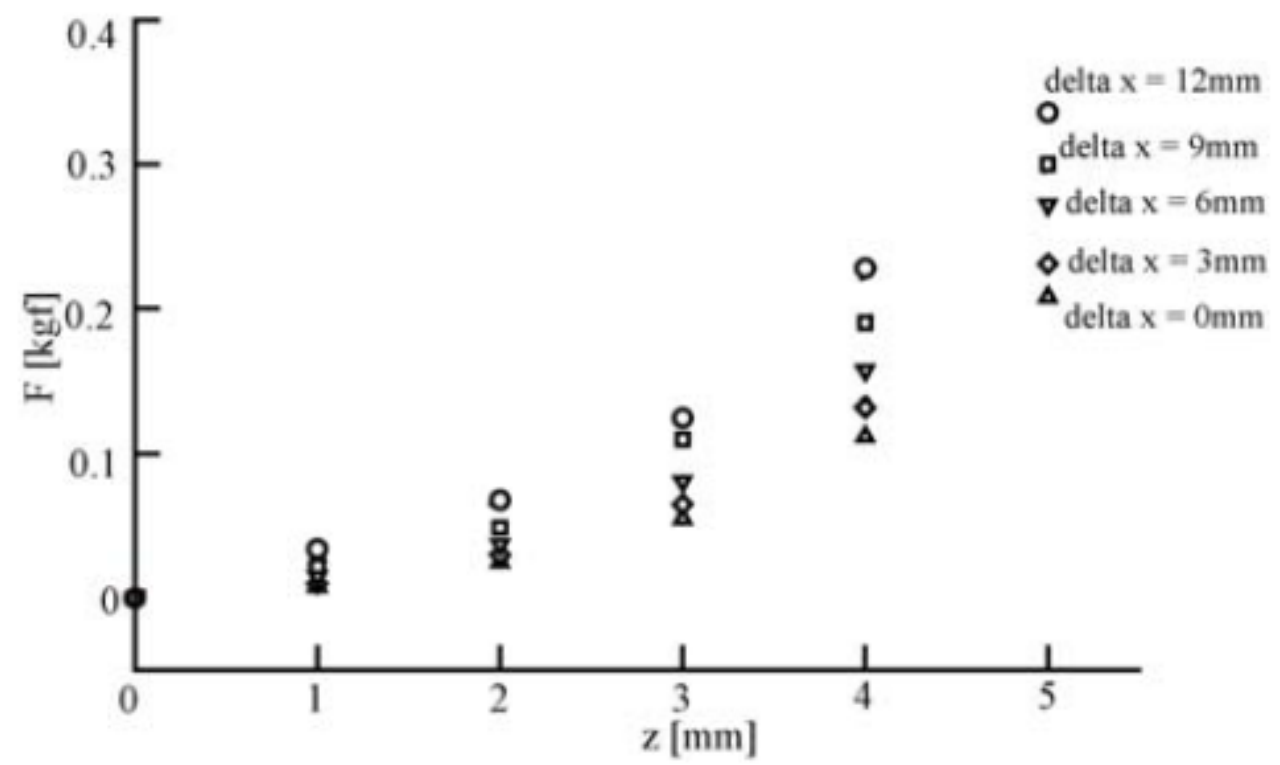

Figure 5. Push length along $z$ axis and $\mathrm{F}$

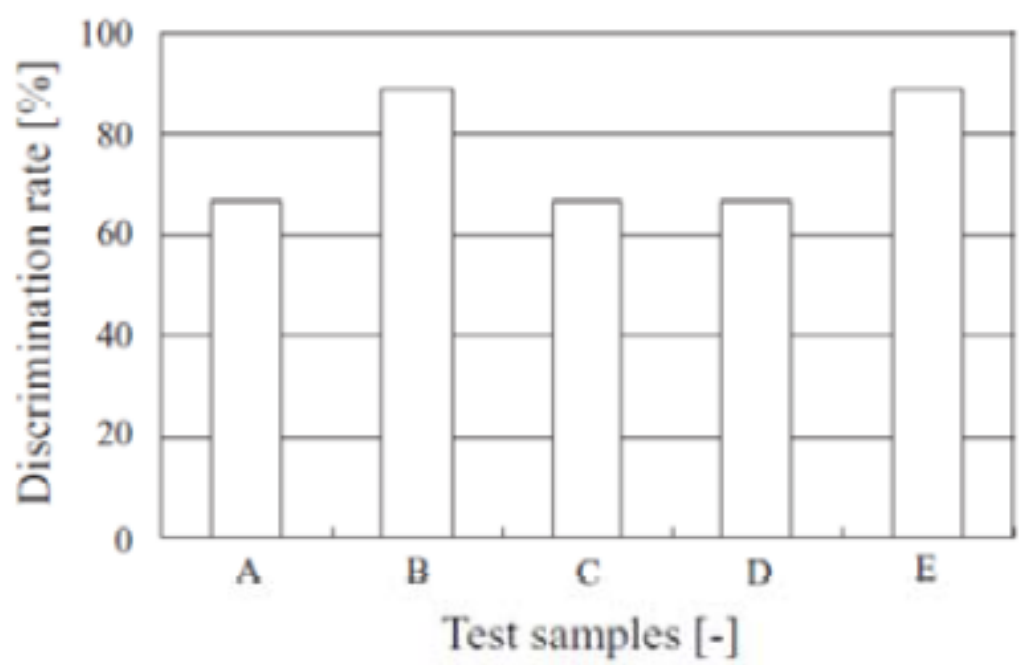

Figure 6. Result of the softness detection ratio 


\section{Softness Control}

The discrimination test has been conducted to verify the sense of touch changed by the proposed principle. In the examination, subjects identified the difference of softness by a different compression distance. Compression distance was settled as 0.0, 6.0, 12.0 $[\mathrm{mm}]$ and we call them as Soft, Medium, and Hard. Two were chosen at random from among three compression distances. Subjects pressed the center part of the cell in the depth of about $2.0[\mathrm{~mm}]$ with the forefinger, and the difference of softness was identified. At all the examinations, finger of the subjects were covered with the cloth. Fig. 6 shows the identification rate by five subjects (A-E). We use three different stiffness values by the $\mathrm{x}$ axis control. Subjects detect the difference of the two different stiffness only use tactile sensation. The average of the identification rate was 76 [\%]. It is thought that the error factor originates in the difference of man's perception characteristic.

\section{Robustness of the Softness Control}

Setups. Fig. 7 shows an experimental setup of the robustness verification. The following physiological experiments were conducted to examine the of human finger tip hardness perception in different materials. The differences in the materials used are the thickness of their elastic part which is $\epsilon_{1}=0.1, \epsilon_{2}=2$ and $\epsilon_{3}=5[\mathrm{~mm}]$. Then, we examined whether all material with different thickness of elastic part can be sensed perfectly when such factors like left/right hand and finger shape when you touch the materials.

Subjects touched two materials' surface using index finger tip and pushed at same deepness $(1[\mathrm{~mm}])$ in the same period of time $(5[\mathrm{~s}])$. When subjects touched and pushed the materials, subjects using two kinds of finger shapes which are normal shape and horizontal shape as shown in Fig. 7(iii). When subjects touched the material's surface with hand's finger the other hand is not working on anything.

Then subjects answered whether they felt any differences in hardness between the two materials. The two materials were randomly picked form the combination of three materials. 
Displacement sensor (to measure the length aling $z$ axis)

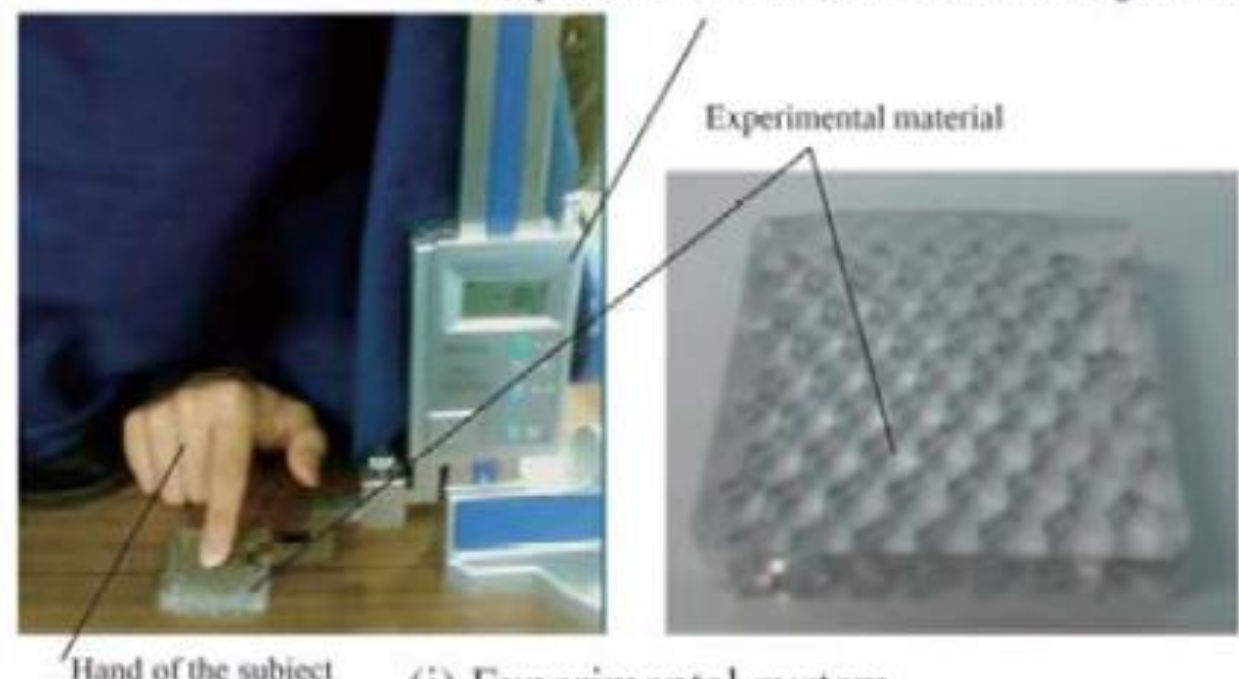

(i) Experimental system
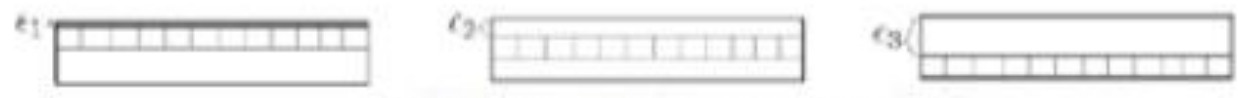

(ii) Three different materials
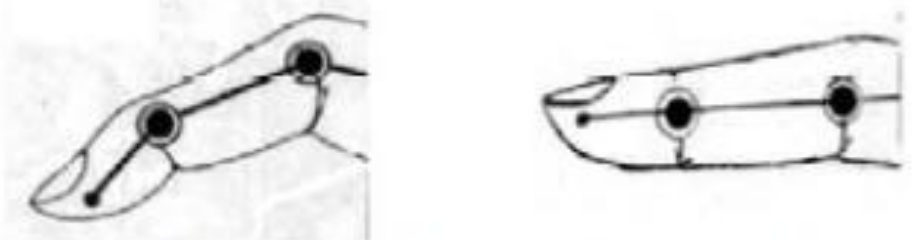

(iii) Normal shape and horizontal shape of the finger tip

Figure 7. Experimental setups of the robustness verification

Results. Fig. 8 (i) shows percentage of success between hands and (ii) shows percentage of success between materials and finger shapes. Fig. 9 shows success rate of the softness detection. Horizontal finger shape is harder shape to produce force to the material can be explained by assuming human finger as a three-link planar manipulator model.

Between the shapes of finger, which are normal shape and horizontal shape; there is significant difference in percentage of success between right and left hand. 


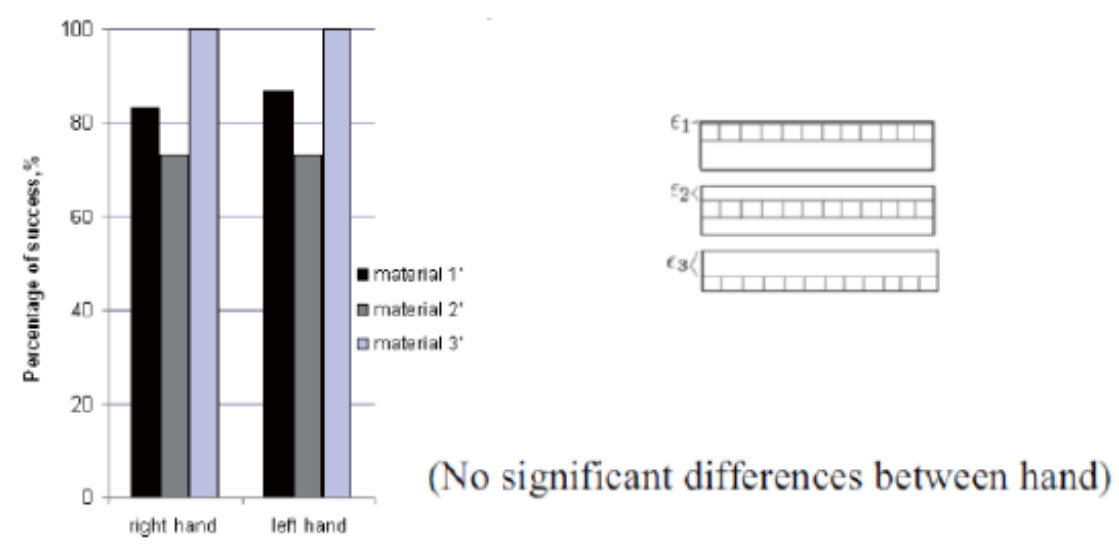

(i) Percentage of success between hands

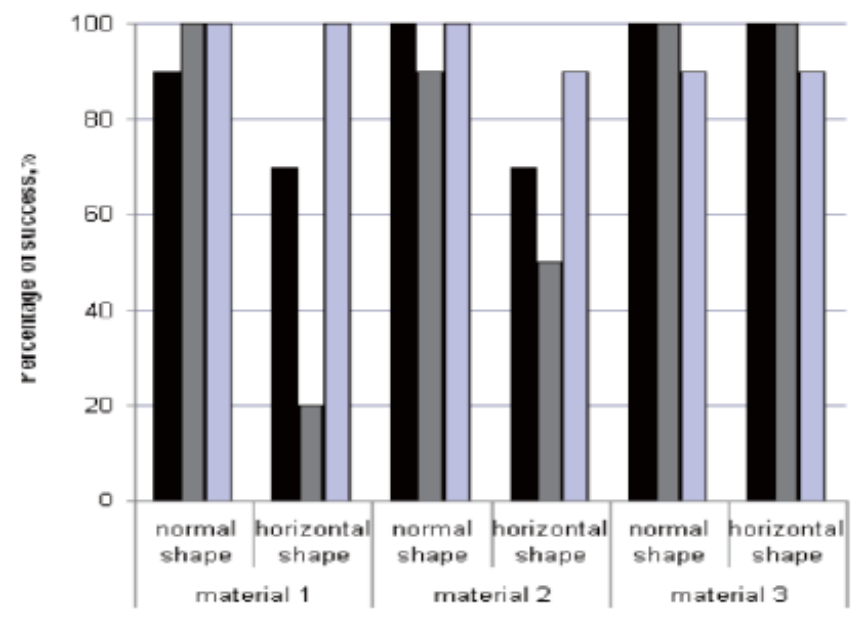

Material $1 \& 2$

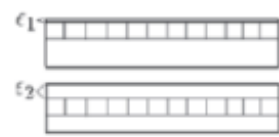

Normal shape $=90$ to $100 \%$

Horizontal shape $=20$ to $70 \%$

Material 3

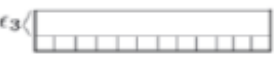

Both shape $=90$ to $100 \%$

(ii) Percentage of success between materials and finger shapes

Figure 8. Experimental results of the robustness verification

Discussion. Fig. 8 (i) shows percentage of success between hands and (ii) shows percentage of success between materials and finger shapes. There is no significant difference in percentage of success between right and left hand. But between shapes of finger, which are normal shape and horizontal shape, there is significant difference. When horizontal shape was used, the success rate to sense the different in hardness between materials decrease especially in sensing materials between material 1 and 2 . However, when comparing material 3 to other materials, subject can sensed the hardness difference almost perfectly for both shape.

Fig. 9 shows success rate of the softness detection. Horizontal finger shape is harder shape to produce force to the material can be explained by assuming human finger as a three-link planar manipulator model. Therefore the harder force can be produced the harder the different in hardness between two materials can be sensed. Which mean finger shape effects the hardness perception in material-1 and material-2. But in material 3 which has the most volume of elastic part case, the required force produced to sense the hardness of the material is the smallest when compared to required force material 1 and 2. Even though the shape of finger was changed, we assumed that the difference between force produce by Normal and Horizontal finger shape is small enough and gives no significant difference in ability to sense hardness. Thinking about 
the Jacobian from three-link planar manipulator, we know that Normal shape can produce force easier than Horizontal shape.
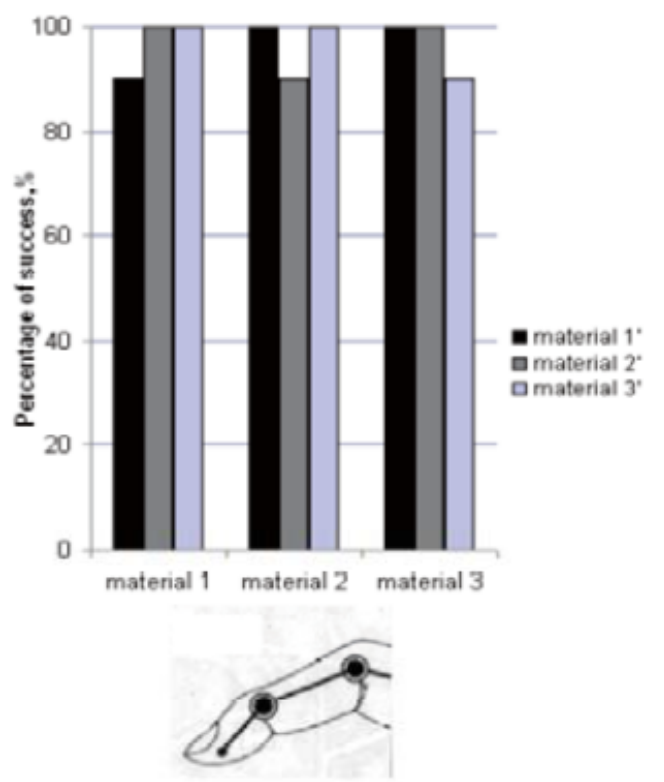

(i) Normal shape
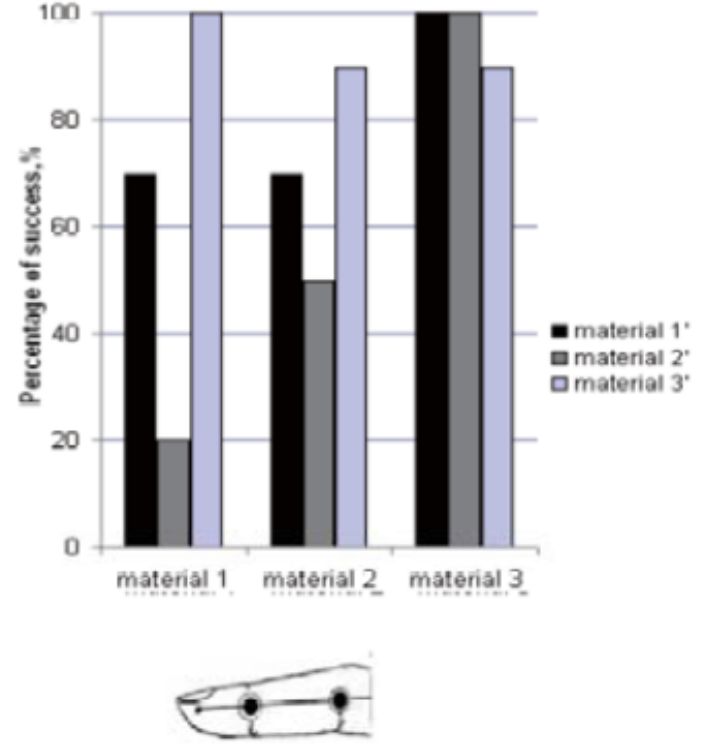

(ii) Horizontal shape

Figure 9. Percentage of success to sense hardness perception

\section{Conclusion}

In this paper, we proposed a principle that used a core material and a flexible resin of the mesh as a presentation display of softness. In the proposed procedure, the adjustment of softness was achieved by a compression of the side of the material. In a basic examination, it was confirmed that the surface trait of the display changed by compression. Then, robustness of the material structure was examined. From these results, softness perception was changed by the differences of the thickness of the elastic parts. In addition, softness detection skill was changed by the posture of the finger. From these results, it is difficult to say the correct structure of the device. But, these results suggest that, we can construct various thicknesses of the elastic parts of the display for the practical requirements. On the other hand, it becomes important to consider about the posture of the users' finger. If at most users will touch the display by normal shape, we can choose large range of the thickness of the elastic parts. Other case, we have to develop a large thickness value.

The improvement of the device that considers a just noticeable difference who corresponds to the flexibility change etc. local, adjusts of the frictional property on the surface, and is human etc. will be advanced in the future. Then, we will extend the method to use them together with the other kind of devices such as the wearable type devices $[15,16,20]$ in order to realize an effective full body display.

\section{Acknowledgement}

This research was financially supported by JSPS Grant in Aid for Scientific Research. 


\section{References}

[1] S. Tachi, T. Maeda, R. Hirata, H. Hoshino, “A construction Method of Virtual Haptic Space", Proc. ICAT 1994, pp. 131--138, 1994.

[2] Hiroshi Hoshino, Susumu Tachi: “'A Method to Represent an Arbitrary Surface in Encounter Type Shape Representation System", Trans. the VirtualReality Society of Japan, vol. 4, no. 2, pp. 445--454, 1999. (in Japanese)

[3] M. Sakaguchi, K. Fukusumi, J. Furusho, “'Development and basic experiments of passive force display using ER brakes", Proc. Japan-USA Symposium on Flexible Automation 2000, 2000.

[4] J. E. Colgate, "Nonholonomic Haptic Display", Proc. ICRA 1996, pp. 539--544, 1996.

[5] M. A. Srinivasan, R. H. Lamotte, "Tactual Discrimination of Softness", Journal of Neurophysiology, vol. 73, no. 1, pp. 88-101, 1995.

[6] A. Bicci, D. E. De Rossi, E. P. Scilingo, “'The role of the contact area spread rate in haptic discrimination of softness", IEEE Trans. on Robotics and Automation, vol. 16, no. 5, pp. 305-310, 2000.

[7] K. Fujita, H. Ohmori, H. Katagiri, “Deveropment of Softness Display Device Based on Fingertip Contact Area Control", Proc. the VirtualReality Society of Japan 5th Annual Conference, pp. 251--254, 2000. (in Japanese)

[8] K. Fujita, H. Ohmori, “A new softness display interface by dynamic fingertip contact area control", Proc. WMSCI 2008, pp. 4229--4235, 2002.

[9] R. Kawamura, H. Yano, H. Iwata, 'Deveropment of surface type haptic interface for presentation of rigidity distribution", Proc. the VirtualReality Society of Japan 5th Annual Conference, pp. 51-54, 2000. (in Japanese)

[10] K. Inoue, R. Uesugi, T. Arai, Y. Mae, 'Development of Haptic Device Using Flexible Sheet", Journal of Robotics and Mechatronics, vol. 15, no. 2, pp. 121-127, 2003.

[11] M. Bianchi, E. P. Scilingo, A. Serio, A. Bicchi, “'A new softness display based on bi-elastic fabric", Proc. of the Third Joint Eurohaptics Conference and Symposium on Haptic Interfaces for Virtual Environment and Teleoperator Systems, pp. 382-383, 2009.

[12] H. Iwata, H. Yano, F. Nakaizumi, R. Kawamura, “Project FEELEX: Adding Haptic Surface to Graphics", Proceeding of SIGGRAPH2001, 2001

[13] H. Iwata, H. Yano, R. Kawamura, “Array force display for hardness distribution", Proceedings 10th Symposium on Haptic Interfaces for Virtual Environment and Teleoperator Systems, 2002

[14] T. Mitsuda, S. Kuge, M. Wakabayashi, S. Kawamura, "Haptic display implemented by controllable passive elements", Proc. ICRA 2002, pp. 4223--4228, 2002.

[15] S. Kawamura, T. Yamamoto, D. Ishida, Y. Nakayama, O. Tabata, S. Sugiyama, "Development of Passive Elements with Variable Mechanical Impedance for Wearable Robots", Proc. ICRA 2002, pp. 248--253, 2002. 
[16] Christopher R. Wagner, S. J. Lederman, Robert D. Howe, “Design And Performance of a Tactile Shape Display Using RC Servomotors", Haptics-e, vol. 3, pp-2004, 2003

[17] R. Ozawa, and H. Kobayashi, “'A new impedance control concept for elastic joint robots", Proc. of the IEEE ICRA2003, Taipei, Taiwan, 2003.

[18] R. Ishibashi, R. Ozawa and S. Kawamura, "Mass estimation in microgravity with a variable stiffness mechanism", Proc. of the IEEE/ASME AIM2017, Zurich, Swiss, 2007.

[19] R. Ishibashi, Muhammad Aliff Bin Rosly, A. Sano, H. Fujimoto, “'A proposal of softness display using honeycomb", ICICIS2014, 2014

[20] M. Kawai, T. Yoshikawa, "Haptic Display of Movable Virtual Object with Interface Device Capable of Continuous-Time Impedance Display by Analog Circuit", Proc. ICRA 2002, pp. 229--234, 2002.

[21] S. Arimoto, “Control theory of nonlinear mechanical systems", Oxford press, 1996. 